\title{
Are Journal Articles Accessed More Times Also Cited More?
}

\author{
Jang Hyun Lee \\ Deputy Editor, Archives of Plastic Surgery \\ Department of Plastic and Reconstructive Surgery, Hanyang University Guri Hospital, Hanyang University \\ College of Medicine, Guri, Korea
}

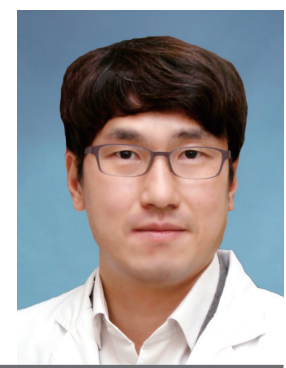

CrossRef reports what is called resolutions every month. This document reports statistics complied monthly on the papers of each journal accessed through the digital object identifier (DOI) proxy server (http://dx.doi.org/). The Archives of Plastic Surgery (APS) also receives the report about which papers are accessed frequently through the DOI system. However, the report only includes papers that are accessed through the DOI proxy server; that is, only those papers accessed by clicking DOI addresses or by entering DOI addresses of the specific articles are included, not the papers looked up by clicking their titles on journal websites or identified through searches of databases such as PubMed, ScienceDirect, and Ovid. Therefore, the resolution report does not fully reflect how many times each paper was accessed. Rather, it indirectly demonstrates that papers are likely to have been accessed often in general when they have been accessed often by DOI search.

We analyzed a total of 320 articles with assigned DOIs published in APS during a two-year period (2012-2013) to determine how frequently they were accessed through the DOI proxy server from January 2012 to March 2014. We found the following: One paper was accessed more than 199 times; four papers 100-199 times; 6 papers $50-99$ times; 22 papers $30-49$ times; and 106 papers $1-29$ times. Thus, 139 papers were accessed at least once. Of the 33 papers that were accessed 30 or more times, original articles and reconstruction were the most common article type and topic, respectively (Tables 1,2 ). We also examined how many times the 139 articles accessed using DOIs at least once during the same period received citations in Science Citation Index (Expanded) (SCI[E]) papers. We found that a total of 62 articles were cited at least once-among them, one was cited more than nine times; eight were cited five to nine times; seven were cited four times; eight were cited three times; eight were cited two times; and 30 were cited once. Of these papers, the 32 papers cited at least twice were also classified by article type and topic, demonstrating that the most frequently cited article type and article topic were original article and reconstruction, respectively (Tables 1,2). Therefore, the type and topic of the most frequently accessed and cited articles tended to be the same. We then looked for a relationship between the number of times the 139 papers accessed at least once by DOI had been accessed and cited, in order to determine whether frequently accessed papers were also frequently cited. We conducted a correlation analysis on the access count and citation count using PASW ver. 18.0 (SPSS Inc., Chicago, IL, USA). We considered a P-value $<0.05$ to indicate statistical significance.

Table 1. Analysis of the type of articles accessed $\mathbf{3 0}$ or more times through the DOI proxy server and cited at least 2 times in $\mathrm{SCI}(\mathrm{E})$ or other journals

\begin{tabular}{|lcc|}
\hline Article type & $\begin{array}{c}\text { No. of articles accessed } \\
30 \text { or more times }\end{array}$ & $\begin{array}{c}\text { No. of articles cited } \\
\text { at least twice }\end{array}$ \\
\hline Original article & 16 & 19 \\
Image & 5 & - \\
Review article & 4 & 6 \\
Case report & 4 & 5 \\
Editorial & 2 & - \\
CME & 1 & 1 \\
Letter & 1 & - \\
Topic & - & 1 \\
Total & 33 & 32 \\
\hline DOI, digital object identifier; SCl(E), Science Citation Index (Expanded); CME, \\
continuing medical examination.
\end{tabular}


Table 2. Analysis of the topic of articles accessed 30 or more times through the digital object identifier (DOI) proxy server and cited at least 2 times in Science Citation Index (Expanded) or other journals

\begin{tabular}{|lcc|}
\hline Article topic & $\begin{array}{c}\text { No. of articles accessed } \\
30 \text { or more times }\end{array}$ & $\begin{array}{c}\text { No. of articles cited } \\
\text { at least twice }\end{array}$ \\
\hline Reconstruction & 10 & 11 \\
Basic & 7 & 9 \\
Tumor & 6 & 2 \\
Cosmetic & 3 & 3 \\
Craniofacial & 3 & 5 \\
Hand & 2 & 1 \\
Other & 2 & 1 \\
Total & 33 & 32 \\
\hline
\end{tabular}

Our analysis identified no correlation between the two variables (correlation coefficient $=0.118, \mathrm{P}=0.165$ ).

Most would agree that enhancing the accessibility and expanding the readership of articles published in journals would improve the probability of their citation $[1,2]$. In general, in terms of the article type and topic, respectively, review articles are more citable than case reports and basic medical research is more citable than clinical medicine. Furthermore, papers that address controversial issues are more likely to be cited than those with topics contributing more broadly to scientific advancement [3]. However, when it comes to simply being widely downloaded and read, the special "Ideas and Innovations" article type (which can be helpful in performing surgery on unique and rare cases) rather than review articles, and articles on clinical medicine (which are more accessible to working clinicians) rather than basic medical research have more practical value. Furthermore, it seems likely that the readership of clinical journals would also like to read articles with a clear takehome message and defined topic rather than those addressing controversial issues, given that the latter are not as immediately applicable to clinical practice. In fact, three of the five most accessed APS papers were case reports (the other two were original articles). On the other hand, four of the six most cited were review articles. This indicates that the topics of articles that were widely read and those that were widely cited were different. This phenomenon has been noted previously, and, accordingly, some have suggested that download counts be used as an alternative for gauging journal quality, in addition to the impact factor, which is currently the primary approach to evaluating the quality of a journal [4]. It seems reasonable to assume that articles downloaded many times can generally be regarded to be of high practical value given that their content has gained a great deal of attention from the readership.

Thus, are journal articles that are accessed more times also cited more often? The answer is "No".

\section{REFERENCES}

1. Brink PA. Article visibility: journal impact factor and availability of full text in PubMed Central and open access. Cardiovasc J Afr 2013;24:295-6.

2. Ali Jawaid S. Striving for improved visibility and increased citation through coverage by PubMed Central (PMC). Pak J Med Sci 2014;30:1-2.

3. Shanta A, Pradhan AS, Sharma SD. Impact factor of a scientific journal: is it a measure of quality of research? J Med Phys 2013;38:155-7.

4. Elliott DB. The impact factor: a useful indicator of journal quality or fatally flawed? Ophthalmic Physiol Opt 2014; $34: 4-7$.

Correspondence: Jang Hyun Lee

Department of Plastic and Reconstructive Surgery, Hanyang University Guri Hospital, Hanyang

University College of Medicine, 153 Gyeongchun-ro, Guri 471-701, Korea

Tel: +82-31-560-2330, Fax: +82-31-560-2338, E-mail: pslee@ hanyang.ac.kr

No potential conflict of interest relevant to this article was reported.

Received: 22 Apr $2014 \bullet$ Revised: 24 Apr 2014 • Accepted: 24 Apr 2014 pISSN: 2234-6163 • elSSN: 2234-6171

http://dx.doi.org/10.5999/aps.2014.41.3.199・ Arch Plast Surg 2014;41:199-200 